\title{
$S$-derivative of perturbed mapping and solution mapping for parametric vector equilibrium problems
}

Xicai Deng ${ }^{1 *}$ and Wei Zhao ${ }^{2}$

"Correspondence:

iamdengxicai@163.com

1 Department of Mathematics and Computer, Guizhou Normal College, 550018, Guiyang, Guizhou, China Full list of author information is

available at the end of the article

\section{Springer}

\begin{abstract}
In this paper, we deal with the sensitivity analysis in vector equilibrium problems by using the $S$-derivative of a set-valued mapping. We first investigate the $S$-derivative on a kind of set-valued gap function for the vector equilibrium problems. Based on these results, S-derivative estimations on a perturbed mapping for the parametric vector equilibrium problem are given. Moreover, we provide some examples to illustrate the obtained results. Finally, we derive the $S$-derivative estimations of a solutions mapping of the parametric vector equilibrium problems via S-derivative estimations of a kind of the parametric variational system.
\end{abstract}

MSC: 49K40; 49J52; 90C29; 90C31

Keywords: Parametric vector equilibrium problems; Set-valued gap function; Perturbed mapping; Solution mapping; S-derivative; Sensitivity analysis

\section{Introduction}

Stability analysis and sensitivity analysis have important theory and application in optimization theory. Roughly speaking, stability analysis investigates the continuity of a perturbation mapping or marginal function of optimization problems. However, sensitivity analysis mainly studies the derivative and subdifferential expressions of a perturbation mapping for optimization problems.

In the past decades, many scholars have carried out extensive research on the sensitivity analysis of numerical optimization problems and obtained some research results, see [16]. It is well known that the optimal value for a vector optimization problem is not unique. Thus, various derivatives of set-valued mappings are usually applied to study derivatives and differential expression of perturbation maps on vector optimization problems, see [712]. On the one hand, the generalized derivatives and coderivatives for set-valued mappings have been used to study the sensitivity analysis of vector optimization problems. Tanino $[13,14]$ studied the behavior of set-valued perturbation maps via the concept of contingent derivative. Kuk, Tanino, and Tanaka $[15,16]$ further investigated sensitivity analysis in vector optimization problems and extended Tanino's results. Especially, Shi $[17,18]$ investigated various sensitivity analysis results in vector optimization problems

(c) The Author(s) 2021. This article is licensed under a Creative Commons Attribution 4.0 International License, which permits use, sharing, adaptation, distribution and reproduction in any medium or format, as long as you give appropriate credit to the original author(s) and the source, provide a link to the Creative Commons licence, and indicate if changes were made. The images or other third party material in this article are included in the article's Creative Commons licence, unless indicated otherwise in a credit line to the material. If material is not included in the article's Creative Commons licence and your intended use is not permitted by statutory regulation or exceeds the permitted use, you will need to obtain permission directly from the copyright holder. To view a copy of this licence, visit http://creativecommons.org/licenses/by/4.0/ 
using the concept of $T P$-derivative which is called $S$-derivative by Chuong [19]. The recent paper provides formulae for inner and outer evaluating of the $S$-derivative of the efficient point multifunction in parametric vector optimization problems. On the other hand, many scholars have studied sensitivity analysis of vector variational inequalities and vector equilibrium problems by using the concept of contingent derivatives introduced by Aubin [20]. Li et al. [21-24] investigated sensitivity analysis of vector variational inequalities by virtue of a set-valued gap function of parametric vector variational inequalities. $\mathrm{Li}$ and $\mathrm{Li}$ [25] obtained some results on sensitivity analysis via a set-valued gap function of parametric vector equilibrium problems. To the best of our knowledge, there have been no results in the literature on sensitivity analysis of a vector equilibrium problem via $S$-derivative of a set-valued mapping, and this is the motivation for our present work.

In this work, the $S$-derivative and the set-valued gap function are exploited to study the sensitivity analysis of vector equilibrium problems. That is, we establish formulae for the $S$-derivative of a perturbation mapping and solution mappings on parametric vector equilibrium problems. The rest of the paper is organized as follows. In Sect. 2, we first provide some basic definitions and notations from vector optimization and set-valued analysis. In Sect. 3, we establish formulae of the $S$-derivative for a set-valued gap function of the parametric vector equilibrium problems. In Sect. 4 , the formulae of $S$-derivative on perturbation mappings for the vector equilibrium problems are given by using the formulae of $S$-derivative in Sect. 3. Moreover, some examples are also simultaneously provided to analyze and illustrate the obtained results. In Sect. 5, the formulae of $S$-derivative on a solution mapping for the parametric vector equilibrium problems is further given under some conditions.

\section{Preliminaries}

Throughout this paper, unless otherwise specified, let $P, X$, and $Y$ be three Euclidean spaces with the usual norms. Let $C$ denote a nonempty, closed, convex, and pointed cone in $Y$ with apex at the origin and int $C \neq \emptyset$, where int $C$ denotes the topological interior of $C$. Furthermore, the origins of all Euclidean spaces are denoted by 0 , the set of nonnegative real numbers is denoted by $\mathbb{R}+$, the set of the positive integer number is denoted by $\mathbb{N}$.

Definition 2.1 ([26]) We say that $y \in \Omega \subset Y$ is called a $C$-minimal point of $\Omega$ iff $(\Omega-$ $\{y\}) \cap(-C)=\{0\}$. The set of all $C$-minimal points of $\Omega$ is denoted by $\min _{C} \Omega$. An element $y \in \Omega \subset Y$ is called a weakly $C$-minimal point of $\Omega$ iff $(\Omega-\{y\}) \cap(-\operatorname{int} C)=\emptyset$. The set of all weakly $C$-minimal points of $\Omega$ is denoted by $\min _{\text {int } C} \Omega$.

Let $F: P \rightarrow 2^{Y}$ be a set-valued mapping. The effective domain and the graph of $F$ are defined by

$$
\begin{aligned}
& \operatorname{dom} F:=\{p \in P \mid F(p) \neq \emptyset\}, \\
& \operatorname{gph} F:=\{(p, y) \in P \times Y \mid y \in F(p)\} .
\end{aligned}
$$

Definition 2.2 ([17]) Let $(\bar{p}, \bar{y}) \in \operatorname{gph} F$.

(i) The $T P$-cone to $\operatorname{gph} F$ at $(\bar{p}, \bar{y})$ is defined by

$$
\begin{aligned}
T P(\operatorname{gph} F ;(\bar{p}, \bar{y})):= & \left\{(p, y) \in P \times Y \mid \exists\left\{t_{n}\right\} \subset \mathbb{R}_{+}, p_{n} \subset P, \exists y_{n} \in F\left(p_{n}\right)\right. \\
& \text { such that } \left.p_{n} \rightarrow \bar{p}, t_{n}\left(p_{n}-\bar{p}, y_{n}-\bar{y}\right) \rightarrow(p, y)\right\} .
\end{aligned}
$$


(ii) The set-valued mapping $D^{S} F(\bar{p}, \bar{y}): P \rightarrow 2^{Y}$ is said to be the $S$-derivative of $F$ at $(\bar{p}, \bar{y})$ iff $\operatorname{gph} D^{S} F(\bar{p}, \bar{y})=T P(\operatorname{gph} F ;(\bar{p}, \bar{y}))$. Equivalently, $\forall p \in P$,

$$
\begin{aligned}
T P(\operatorname{gph} F ;(\bar{p}, \bar{y})):= & \left\{(p, y) \in P \times Y \mid \exists\left\{t_{n}\right\} \subset \mathbb{R}_{+}, p_{n} \subset P, \exists y_{n} \in F\left(p_{n}\right)\right. \\
& \text { such that } \left.p_{n} \rightarrow \bar{p}, t_{n}\left(p_{n}-\bar{p}, y_{n}-\bar{y}\right) \rightarrow(p, y)\right\} .
\end{aligned}
$$

Definition 2.3 ([27]) The domination property holds for $F$ around $\bar{p} \in P$ iff there exists a neighborhood $U$ of $\bar{p}$ such that

$$
F(p) \subset \min _{C} F(p)+C, \quad \forall p \in U
$$

We consider the following parametric vector equilibrium problem: find $x \in K(p)$ such that

$$
f(p, x, y) \notin-C \backslash\{0\}, \quad \forall y \in K(p),
$$

where $x$ is a decision variable, $p$ is a perturbation parameter, $f: P \times X \times X \rightarrow Y$ is a vectorvalued objective function, and $K: P \rightarrow 2^{X}$ is a set-valued constraint map, which specifies a feasible decision set. The parametric vector optimization problem is a kind of special case of the parametric vector equilibrium problem. For all $x, y \in K(p)$, we take

$$
f(p, x, y):=h(p, y)-h(p, x)
$$

where $h: P \times X \rightarrow Y$ is a vector-valued mapping. Then we may get the parametric vectorvalued optimization problem:

$$
\min _{C} h(p, y), \quad \forall y \in K(p)
$$

We now introduce a class of set-valued gap functions for parametric vector equilibrium problems. Define a set-valued mapping $G: P \times X \rightarrow 2^{Y}$ by

$$
G(p, x)=\bigcup_{y \in K(p)} f(p, x, y) \cup\{0\}
$$

A set-valued mapping $V: P \times X \rightarrow 2^{Y}$ is defined by

$$
V(p, x)=\min _{C} G(p, x), \quad \forall(p, x) \in g p h K .
$$

Proposition 2.1 The set-valued mapping $V$ is a gap function of parametric vector equilibrium problems.

Proof Take any $z \in C \backslash\{0\}, x \in K(p)$ and $\left.0 \in G_{(p}, x\right)$, then we have

$$
0 \in\{z-C \backslash\{0\}\} \cap G(p, x) .
$$

Thus, $z \notin V(p, x)$ and then $V(p, x) \cap\{-C \backslash\{0\}\}=\emptyset$, which shows that $0 \in V(p, x)$. 
Using the definition of $V$, we have

$$
0 \in V(p, x) \quad \Leftrightarrow \quad 0 \in \min _{C} G(p, x) \quad \Leftrightarrow \quad G(p, x) \cap\{-C\}=\{0\}
$$

So we get $G(p, x) \cap\{-C \backslash\{0\}\}=\emptyset$, that is, $f(p, x, y) \notin-C \backslash\{0\}, \forall y \in K(p)$.

We give an example on the class set-valued gap functions for parametric vector equilibrium problems.

Example 2.1 Let $P=X=Y=\mathbb{R}$ and $C=\mathbb{R}_{+}$. Putting $f(p, x, y):=x(p+y)$ and $K(p):=$ $[-|p|, p]$. So we get

$$
G(p, x)=\bigcup_{y \in K(p)} x(p+y) \cup\{0\} .
$$

Hence,

$$
V(p, x)= \begin{cases}\{2 p x\}, & \text { if } p>0, x<0 \\ \{0\}, & \text { otherwise }\end{cases}
$$

It is easy to check that (1) becomes set-valued gap functions for the above parametric vector equilibrium problems.

\section{S-derivative of set-valued gap functions for parametric vector equilibrium problems}

In this section, we derive the formulas for computing $S$-derivative of the set-valued gap function $V$ for parametric vector equilibrium problems. We first need to compute $S$ derivative of the set-valued mapping $G+C$.

Proposition 3.1 Let $(\bar{p}, \bar{x}, \bar{z}) \in \operatorname{gph} G$. Assume that

$$
D^{S} G(\bar{p}, \bar{x}, \bar{z})(0,0) \cap(-C)=\{0\} .
$$

One has

$$
D^{S} G(\bar{p}, \bar{x}, \bar{z})(p, x)+C=D^{S}(G+C)(\bar{p}, \bar{x}, \bar{z})(p, x), \quad \forall(p, x) \in P \times X .
$$

Proof Let us first prove that $D^{S} G(\bar{p}, \bar{x}, \bar{z})(p, x)+C \subset D^{S}(G+C)(\bar{p}, \bar{x}, \bar{z})(p, x)$ for all $(p, x) \in$ $P \times X$. For any $p \in P, z \in D^{S} G(\bar{p}, \bar{x}, \bar{z})(p, x)$ and $c \in C$, then there are sequences $\left\{t_{n}\right\} \subset \mathbb{R}_{+}$, $\left\{\left(p_{n}, x_{n}\right)\right\} \subset P \times X$ and $\left\{z_{n}\right\} \subset Y$ such that

$$
z_{n} \in G\left(p_{n}, x_{n}\right), \quad\left(p_{n}, x_{n}\right) \rightarrow(\bar{p}, \bar{x}), \quad t_{n}\left(p_{n}-\bar{p}, x_{n}-\bar{x}, z_{n}-\bar{z}\right) \rightarrow(p, x, z) .
$$

It follows that

$$
z_{n}+\frac{1}{t_{n}} c \in G\left(p_{n}, x_{n}\right)+C, \quad\left(p_{n}, x_{n}\right) \rightarrow(\bar{p}, \bar{x}),
$$




$$
t_{n}\left(p_{n}-\bar{p}, x_{n}-\bar{x}, z_{n}+\frac{1}{t_{n}} c-\bar{z}\right) \rightarrow(p, x, z+c)
$$

Thus, $z+c \in D^{S}(G+C)(\bar{p}, \bar{x}, \bar{z})(p, x)$. Now we justify the reverse inclusion. For all $z \in D^{S}(G+$ $C)(\bar{p}, \bar{x}, \bar{z})$, there are sequences $\left\{t_{n}\right\} \subset \mathbb{R}_{+},\left\{\left(p_{n}, x_{n}\right)\right\} \subset P \times X,\left\{z_{n}\right\} \subset Y$ and $\left\{c_{n}\right\} \subset C$ such that

$$
z_{n} \in G\left(p_{n}, x_{n}\right), \quad\left(p_{n}, x_{n}\right) \rightarrow(\bar{p}, \bar{x}), \quad t_{n}\left(p_{n}-\bar{p}, x_{n}-\bar{x}, z_{n}+c_{n}-\bar{z}\right) \rightarrow(p, x, z)
$$

If there is $n_{0} \in \mathbb{N}$ such that $c_{n}=0$ for all $n \geq n_{0}$, then $z \in D^{S} G(\bar{p}, \bar{x}, \bar{z})(\bar{p}, \bar{x})$. Otherwise, we may suppose that $c_{n} \neq 0, \forall n \in N$. Assume further that

$$
\lim _{n \rightarrow \infty} \frac{c_{n}}{\left\|c_{n}\right\|}=c \in C \backslash\{0\} .
$$

Next, we prove that the sequence $\left\|t_{n} c_{n}\right\|$ is bounded, if not, assume that $\lim _{n \rightarrow \infty}\left\|t_{n} c_{n}\right\|=$ $+\infty$. It holds that

$$
\frac{1}{c_{n}}\left(p_{n}-\bar{p}, x_{n}-\bar{x}, z_{n}-\bar{z}\right)=\left(\frac{t_{n}\left(p_{n}-\bar{p}\right)}{\left\|t_{n} c_{n}\right\|}, \frac{t_{n}\left(x_{n}-\bar{x}\right)}{\left\|t_{n} c_{n}\right\|}, \frac{t_{n}\left(z_{n}+c_{n}-\bar{z}\right)}{\left\|t_{n} c_{n}\right\|}-\frac{c_{n}}{\left\|c_{n}\right\|}\right) .
$$

Thus, we have

$$
z_{n} \in G\left(p_{n}, x_{n}\right), \quad\left(p_{n}, x_{n}\right) \rightarrow(\bar{p}, \bar{x}), \quad \frac{1}{c_{n}}\left(p_{n}-\bar{p}, x_{n}-\bar{x}, z_{n}-\bar{z}\right) \rightarrow(0,0,-c) .
$$

Therefore, $-c \in D^{S} G(\bar{p}, \bar{x}, \bar{z})(0,0)$, which contradicts (2). So our results are obtained. Without loss of generality, we may suppose that $\left\|t_{n} c_{n}\right\| \rightarrow a>0$. We get

$$
t_{n}\left(p_{n}-\bar{p}, x_{n}-\bar{x}, z_{n}-\bar{z}\right)=\left(t_{n}\left(p_{n}-\bar{p}\right), t_{n}\left(x_{n}-\bar{x}\right), t_{n}\left(z_{n}+c_{n}-\bar{z}\right)-\left\|t_{n} c_{n}\right\| \frac{c_{n}}{\left\|c_{n}\right\|}\right) .
$$

Combining this with (3) and (4), we have

$$
z_{n} \in G\left(p_{n}, x_{n}\right), \quad\left(p_{n}, x_{n}\right) \rightarrow(\bar{p}, \bar{x}), \quad t_{n}\left(p_{n}-\bar{p}, x_{n}-\bar{x}, z_{n}-\bar{z}\right) \rightarrow(p, x, z-a c)
$$

Thus, $z-a c \in D^{S} G(\bar{p}, \bar{x}, \bar{z})(p, x)$. That is, $z \in D^{S} G(\bar{p}, \bar{x}, \bar{z})(p, x)+C$, which completes the proof.

The following example illustrates that the condition in Proposition 3.1 is essential.

Example 3.1 Let $P=X=Y=\mathbb{R}, C=\mathbb{R}_{+}$and $G: P \times X \rightarrow 2^{Y}$ be defined by

$$
G(p, x):= \begin{cases}\{0\}, & \text { if } p+x \leq 0 \\ \{p+x,-\sqrt{p+x}\}, & \text { if } p+x>0 .\end{cases}
$$

Consider $\bar{p}=0, \bar{x}=0$ and $\bar{z}=0$. By computing, we get

$$
D^{S} G(\bar{p}, \bar{x}, \bar{z})(p, x)= \begin{cases}\{0\}, & \text { if } p+x<0 \\ ]-\infty, 0], & \text { if } p+x=0 \\ p+x, & \text { if } p+x>0\end{cases}
$$




$$
D^{S}(G+C)(\bar{p}, \bar{x}, \bar{z})= \begin{cases}\mathbb{R}_{+}, & \text {if } p+x \leq 0, \\ \mathbb{R}, & \text { if } p+x>0\end{cases}
$$

Then

$$
\left.\left.D^{S} G(\bar{p}, \bar{x}, \bar{z})(0,0) \cap(-C)=\right]-\infty, 0\right] .
$$

When $p+x>0$, we have

$$
\begin{aligned}
& D^{S} G(\bar{p}, \bar{x}, \bar{z})(p, x)+C=\mathbb{R}_{+}, \\
& D^{S}(G+C)(\bar{p}, \bar{x}, \bar{z})(p, x)=\mathbb{R} .
\end{aligned}
$$

Hence,

$$
D^{S} G(\bar{p}, \bar{x}, \bar{z})(p, x)+C \neq D^{S}(G+C)(\bar{p}, \bar{x}, \bar{z})(p, x), \quad \text { as } p+x>0 .
$$

Using Proposition 3.1 and reference [19], we give $S$-derivative formulas of $V$ at the reference point via the set of $C$-minimal points or weakly $C$-minimal points of the $S$-derivative of $G$ at the corresponding point.

Theorem 3.1 Let $(\bar{p}, \bar{x}, \bar{z}) \in \operatorname{gph} V$. Assume that $G$ has the domination property around $(\bar{p}, \bar{x})$. Suppose that one of the following conditions is satisfied:

(i) $D^{S} G(\bar{p}, \bar{x}, \bar{z})(0,0) \cap(-C)=\{0\}$;

(ii) $G(p, x)$ is convex for $(p, x) \in U$, where $U$ is a neighborhood of $(\bar{p}, \bar{x})$.

One has

$$
D^{S} V(\bar{p}, \bar{x}, \bar{z})(p, x) \supset \min _{C} D^{S} G(\bar{p}, \bar{x}, \bar{z})(p, x)
$$

Theorem 3.2 Let $(\bar{p}, \bar{x}, \bar{z}) \in \operatorname{gph} V$. Assume for any $(p, x, z) \in \operatorname{TP}(\operatorname{gph} V ;(\bar{p}, \bar{x}, \bar{z}))$ that

$$
\begin{aligned}
D^{S} G(\bar{p}, \bar{x}, \bar{z})(p, x) \cap(z-\operatorname{int} C) \subset\{ & \left\{v \in Z \mid \forall t_{n} \subset R_{+}, \forall\left\{\left(p_{n}, x_{n}\right)\right\} \subset P \times X,\right. \\
& \left(p_{n}, x_{n}\right) \rightarrow(\bar{p}, \bar{x}), t_{n}\left(p_{n}-\bar{p}, x_{n}-\bar{x}\right) \rightarrow(p, x), \\
& \left.\exists \bar{z}_{n} \in G\left(p_{n}, x_{n}\right), t_{n}\left(\bar{z}_{n}-\bar{z}\right) \rightarrow v\right\} .
\end{aligned}
$$

One has

$$
\left.D^{S} V(\bar{p}, \bar{x}, \bar{z})(p, x)\right) \subset \min _{\operatorname{int} C} D^{S} G(\bar{p}, \bar{x}, \bar{z})(p, x), \quad \forall(p, x) \in P \times X
$$

Remark 3.1 We mention that our results in Theorem 3.1 and Theorem 3.2 are new, and therefore they do not coincide with the existing ones in the literature (see [28-30] and the cited references therein). 


\section{S-derivative of perturbation maps for parametric vector equilibrium problems}

In this section, we give a formula for computing the $S$-derivative of the perturbation map in parameterized vector equilibrium problems by using the formulae of $S$-derivative in Sect. 3. Finally we provide an example to analyze and illustrate these results.

Lemma 4.1 Let $\bar{p} \in P, \bar{x} \in X$ and $\bar{y} \in \Omega=\{y \in K(\bar{p}) \mid f(\bar{p}, \bar{x}, y)=0\}$. Assume that $f$ is continuous and Fréchet differentiable at $(\bar{p}, \bar{x}, \bar{y})$. Moreover, $K$ is compact and that

$$
D^{S} K(\bar{p}, \bar{y})(0)=\{0\} .
$$

One has

$$
D^{S} G(\bar{p}, \bar{x}, 0)(p, x)=\bigcup_{\bar{y} \in \Omega}\left\{\nabla f(\bar{p}, \bar{x}, \bar{y})(p, x, y) \mid y \in D^{S} K(\bar{p}, \bar{y})(p)\right\}, \quad \forall(p, x) \in P \times X .
$$

Proof Let us first justify that (7) leads to the following:

$$
D^{S} G(\bar{p}, \bar{x}, 0)(0,0)=\{0\} .
$$

Assume to the contrary that $D^{S} G(\bar{p}, \bar{x}, 0)(0,0) \neq\{0\}$, then there is $z_{0} \in D^{S} G(\bar{p}, \bar{x}, 0)(0,0) \backslash\{0\}$. Hence, there are sequences $\left\{t_{n}\right\} \subset \mathbb{R}_{+},\left\{\left(p_{n}, x_{n}\right)\right\} \subset P \times X$ and $\left\{z_{n}\right\} \subset Y$ such that

$$
z_{n} \in G\left(p_{n}, x_{n}\right), \quad\left(p_{n}, x_{n}\right) \rightarrow(\bar{p}, \bar{x}), \quad t_{n}\left(p_{n}-\bar{p}, x_{n}-\bar{x}, z_{n}\right) \rightarrow\left(0,0, z_{0}\right) .
$$

Thus, if $\left(p_{n}, x_{n}\right)=(\bar{p}, \bar{x})$ for all $n \in \mathbb{N}$, we take $t_{n} \in\left(0, \min \left\{\frac{1}{n}, \frac{\left\|z_{n}\right\|}{n}\right\}\right)$, otherwise $t_{n}=\max \left\{\| p_{n}-\right.$ $\left.\bar{p}\|,\| x_{n}-\bar{x} \|\right\}$. Then $t_{n} \rightarrow 0$ and $\left\|\frac{z_{n}}{t_{n}}\right\| \rightarrow \infty$. By $z_{n} \in G\left(p_{n}, x_{n}\right)$ for all $n \in \mathbb{N}$, there is $\left\{\left(p_{n}, x_{n}\right)\right\} \subset P \times X$ such that

$$
y_{n} \in K\left(p_{n}\right), \quad z_{n}=f\left(p_{n}, x_{n}, y_{n}\right), \quad \forall n \in \mathbb{N} .
$$

Let

$$
\tilde{p}_{n}=\frac{p_{n}-\bar{p}}{t_{n}}, \quad \tilde{x}_{n}=\frac{x_{n}-\bar{x}}{t_{n}}, \quad \tilde{y}_{n}=\frac{y_{n}-\bar{y}}{t_{n}}, \quad \forall n \in \mathbb{N} .
$$

Since $\left\|\tilde{p}_{n}\right\| \leq 1$ and $\left\|\tilde{x}_{n}\right\| \leq 1$ for all $n \in \mathbb{N}$, we may suppose that $\tilde{p}_{n} \rightarrow \tilde{p} \in P$ and $\tilde{x}_{n} \rightarrow \tilde{x} \in X$. We prove that $\left\{\tilde{y}_{n}\right\}$ is bounded, if not, one may assume that $\left\|\tilde{y}_{n}\right\| \rightarrow \infty$. For any $n \in \mathbb{N}$, set

$$
\hat{y}_{n}=\frac{\tilde{y}_{n}}{\left\|\tilde{y}_{n}\right\|}, \quad \hat{p}_{n}=\frac{\tilde{p}_{n}}{\left\|\tilde{y}_{n}\right\|}, \quad \hat{t}_{n}=t_{n}\left\|\tilde{y}_{n}\right\|, \quad \tilde{t}_{n}=\frac{1}{\hat{t}_{n}} .
$$

Then $\hat{t}_{n} \hat{p}_{n}=t_{n} \tilde{p}_{n}, \hat{t}_{n} \hat{y}_{n}=t_{n} \tilde{y}_{n},\left\|\hat{y}_{n}\right\|=1$ for all $n \in \mathbb{N}$ and $\hat{p}_{n} \rightarrow 0$. Since $X$ is finite dimension, we may suppose that $\hat{y}_{n} \rightarrow \hat{y}$ with $\|\hat{y}\|=1$. It is easy to see that

$$
p_{n}=\bar{p}+t_{n} \tilde{p}_{n}=\bar{p}+\hat{t}_{n} \hat{p}_{n}, \quad y_{n}=\bar{y}+t_{n} \tilde{y}_{n}=\bar{y}+\hat{t}_{n} \hat{y}_{n}, \quad \forall n \in \mathbb{N} .
$$


Hence,

$$
\tilde{t}_{n}\left(p_{n}-\bar{p}\right)=\tilde{t}_{n} \hat{t}_{n} \hat{p}_{n}=\hat{p}_{n} \rightarrow 0, \quad \tilde{t}_{n}\left(y_{n}-\bar{y}\right)=\tilde{t}_{n} \hat{t}_{n} \hat{y}_{n}=\hat{y}_{n} \rightarrow \hat{y}
$$

Thus, there are the sequences $\left\{\tilde{t}_{n}\right\} \subset \mathbb{R}_{+},\left\{\left(p_{n}, y_{n}\right)\right\} \subset P \times X$ such that

$$
y_{n} \in K\left(p_{n}\right), \quad p_{n} \rightarrow \bar{p}, \quad \tilde{t}_{n}\left(p_{n}-\bar{p}, y_{n}-\bar{y}\right) \rightarrow(0, \hat{y}) .
$$

This means that $\hat{y} \in D^{S} K(\bar{p}, \bar{y})(0)$, which contradicts (7). So $\left\{\tilde{y}_{n}\right\}$ is bounded. There is no loss of generality in assuming that $\tilde{y}_{n} \rightarrow \tilde{y} \in X$. By (10) and (11),

$$
z_{n}=f\left(\bar{p}+t_{n} \tilde{p}_{n}, \bar{x}+t_{n} \tilde{x}_{n}, \bar{y}+t_{n} \tilde{y}_{n}\right), \quad \forall n \in \mathbb{N} .
$$

Since $f$ is Fréchet differentiable at $(\bar{p}, \bar{x}, \bar{y})$, it holds that

$$
\lim _{n \rightarrow \infty} \frac{z_{n}}{t_{n}}=\lim _{n \rightarrow \infty} \frac{f\left(\bar{p}+t_{n} \tilde{p}_{n}, \bar{x}+t_{n} \tilde{x}_{n}, \bar{y}+t_{n} \tilde{y}_{n}\right)}{t_{n}}=\nabla f(\bar{p}, \bar{x}, \bar{y})(\tilde{p}, x, \tilde{y}),
$$

which contradicts $\left\|\frac{z_{n}}{t_{n}}\right\| \rightarrow \infty$. Thus, (9) holds true. Note that (7) and (9) lead to (8) for $p=0$. Hence, it is enough to prove (8) in the case $p \neq 0$. We now justify the inclusion

$$
D^{S} G(\bar{p}, \bar{x}, 0)(p, x) \supset \bigcup_{\bar{y} \in \Omega}\left\{\nabla f(\bar{p}, \bar{x}, \bar{y})(p, x, y) \mid y \in D^{S} K(\bar{p}, \bar{y})(p)\right\}, \quad \forall p \neq 0
$$

Take any $p \in P \backslash\{0\}$ and $y \in D^{S} K(\bar{p}, \bar{y})(p)$. Set $z:=\nabla f(\bar{p}, \bar{x}, \bar{y})(p, x, y), \forall \bar{y} \in \Omega$. We must show that $z \in D^{S} G(\bar{p}, \bar{x}, 0)(p, x)$. Since $y \in D^{S} K(\bar{p}, \bar{x})(p)$, there are $\left\{t_{n}\right\} \subset \mathbb{R}_{+},\left\{p_{n}\right\} \subset P$ and $y_{n} \in$ $K\left(p_{n}\right)$ for all $n \in \mathbb{N}$ such that

$$
p_{n} \rightarrow \bar{p}, \quad t_{n}\left(p_{n}-\bar{p}, y_{n}-\bar{y}\right) \rightarrow(p, y)
$$

Putting $\tilde{p}_{n}=t_{n}\left(p_{n}-\bar{p}\right)$ and $\tilde{y}_{n}=t_{n}\left(y_{n}-\bar{y}\right)$. Then $p_{n} \rightarrow \bar{p}, \tilde{p}_{n}=t_{n}\left(p_{n}-\bar{p}\right) \rightarrow p$. So $p \neq 0$ and $t_{n}$ is unbounded. We may suppose that $t_{n} \rightarrow \infty$. Setting $\tilde{t}_{n}=\frac{1}{t_{n}}$. Then there are $\left\{\tilde{t}_{n}\right\} \subset \mathbb{R}_{+}$, $\left\{p_{n}\right\} \subset P$ and $\left\{\tilde{y}_{n}\right\} \subset X$ such that

$$
\tilde{t}_{n} \rightarrow 0, \tilde{p}_{n} \rightarrow p, \tilde{y}_{n} \rightarrow y, \quad \bar{y}+\tilde{t}_{n} \tilde{y}_{n} \in K\left(\bar{p}+\tilde{t}_{n} \tilde{p}_{n}\right) .
$$

Set $\tilde{x}_{n}=x$ for all $n \in \mathbb{N}$. By the definition of $G$, we have

$$
z_{n}=f\left(\bar{p}+\tilde{t}_{n} \tilde{p}_{n}, \bar{x}+\tilde{t}_{n} \tilde{x}_{n}, \bar{y}+\tilde{t}_{n} \tilde{y}_{n}\right) \in G\left(\bar{p}+t_{n} \tilde{p}_{n}, \bar{x}+\tilde{t}_{n} \tilde{x}_{n}\right), \quad \forall n \in \mathbb{N} .
$$

Since $f$ is Fréchet differentiable at $(\bar{p}, \bar{x}, \bar{y})$, it holds that

$$
\lim _{n \rightarrow \infty} t_{n} z_{n}=\lim _{n \rightarrow \infty} \frac{f\left(\bar{p}+\tilde{t}_{n} \tilde{p}_{n}, \bar{x}+\tilde{t}_{n} \tilde{x}_{n}, \bar{y}+\tilde{t}_{n} \tilde{y}_{n}\right)}{\tilde{t}_{n}}=\nabla f(\bar{p}, \bar{x}, \bar{y})(p, x, y)=z .
$$

Thus, there are $\left\{t_{n}\right\} \subset \mathbb{R}_{+},\left\{\left(p_{n}, x_{n}\right)\right\} \subset P \times X$ and $z_{n} \in G\left(p_{n}, x_{n}\right)$ such that

$$
\left(p_{n}, x_{n}\right) \rightarrow(\bar{p}, \bar{x}), \quad t_{n}\left(p_{n}-\bar{p}, x_{n}-\bar{x}, z_{n}\right) \rightarrow(p, x, z)
$$

This means that $z \in D^{S} G(\bar{p}, \bar{x}, 0)(p, x)$. 
We now verify the reverse inclusion

$$
D^{S} G(\bar{p}, \bar{x}, 0)(p, x) \subset \bigcup_{\bar{y} \in \Omega}\left\{\nabla f(\bar{p}, \bar{x}, \bar{y})(p, x, y) \mid y \in D^{S} K(\bar{p}, \bar{y})(p)\right\}, \quad \forall p \neq 0
$$

For all $p \in P \backslash\{0\}$, take any $y \in D^{S} G(\bar{p}, \bar{x}, 0)(p, x)$. Then there exist sequences $\left\{t_{n}\right\} \subset \mathbb{R}_{+}$, $\left\{\left(p_{n}, x_{n}\right)\right\} \subset P \times X$ and $z_{n} \in G\left(p_{n}, x_{n}\right)$ for all $n \in \mathbb{N}$ such that

$$
\left(p_{n}, x_{n}\right) \rightarrow(\bar{p}, \bar{x}), \quad t_{n}\left(p_{n}-\bar{p}, x_{n}-\bar{x}, z_{n}\right) \rightarrow(p, x, z)
$$

Set

$$
\tilde{p}_{n}:=t_{n}\left(p_{n}-\bar{p}\right), \quad \tilde{x}_{n}:=t_{n}\left(x_{n}-\bar{x}\right), \quad \forall n \in \mathbb{N} .
$$

Since $\tilde{p}_{n}=t_{n}\left(p_{n}-\bar{p}\right) \rightarrow p \neq 0$, it shows that $\left\{t_{n}\right\}$ is unbounded. Without loss of generality, one may suppose that $t_{n} \rightarrow \infty$. By $z_{n} \in G\left(p_{n}, x_{n}\right)$, there exists $\left\{y_{n}\right\} \subset X$ such that

$$
y_{n} \in K\left(p_{n}\right), \quad z_{n}=f\left(p_{n}, x_{n}, y_{n}\right), \quad \forall n \in \mathbb{N} .
$$

Let $\tilde{y}_{n}=t_{n}\left(y_{n}-\tilde{y}\right)$. We prove that $\left\{\tilde{y}_{n}\right\}$ is bounded. If not, we may assume that $\left\|\tilde{y}_{n}\right\| \rightarrow \infty$. For any $n \in \mathbb{N}$, set

$$
\hat{y}_{n}=\frac{\tilde{y}_{n}}{\left\|\tilde{y}_{n}\right\|}, \quad \hat{p}_{n}=\frac{\tilde{p}_{n}}{\left\|\tilde{y}_{n}\right\|}, \quad \hat{t}_{n}=\frac{\left\|\tilde{y}_{n}\right\|}{t_{n}}, \quad \tilde{t}_{n}=\frac{1}{\hat{t}_{n}} .
$$

Then $\hat{t}_{n} \hat{p}_{n}=\frac{\tilde{p}_{n}}{t_{n}}, \hat{t}_{n} \hat{y}_{n}=\frac{\tilde{y}_{n}}{t_{n}},\left\|\hat{y}_{n}\right\|=1$ for all $n \in \mathbb{N}$ and $\hat{p}_{n} \rightarrow 0$. Since $\left\|\hat{y}_{n}\right\|=1$ and $X$ is finite dimension, we may assume that $\hat{y}_{n} \rightarrow \hat{y}$ and $\|\hat{y}\|=1$. It holds that

$$
p_{n}=\bar{p}+\frac{\tilde{p}_{n}}{t_{n}}=\bar{p}+\hat{t}_{n} \hat{p}_{n}, \quad y_{n}=\bar{y}+\frac{\tilde{y}_{n}}{t_{n}}=\bar{y}+\hat{t}_{n} \hat{y}_{n}, \quad \forall n \in \mathbb{N} .
$$

Hence,

$$
\tilde{t}_{n}\left(p_{n}-\bar{p}\right)=\tilde{t}_{n} \hat{t}_{n} \hat{p}_{n}=\hat{p}_{n} \rightarrow 0, \quad \tilde{t}_{n}\left(y_{n}-\bar{y}\right)=\tilde{t}_{n} \hat{t}_{n} \hat{y}_{n}=\hat{y}_{n} \rightarrow \hat{y}
$$

So, we have the sequences $\left\{t_{n}\right\} \subset \mathbb{R}_{+},\left\{\left(p_{n}, y_{n}\right)\right\} \subset P \times X$ satisfying $y_{n} \in K\left(p_{n}\right)$ for all $n \in \mathbb{N}$ and

$$
p_{n} \rightarrow \bar{p}, \quad \tilde{t}_{n}\left(p_{n}-\bar{p}, y_{n}-\bar{y}\right) \rightarrow(0, \hat{y})
$$

That is, $\hat{y} \in D^{S} K(\bar{p}, \bar{y})(0)$, which contradicts (7). Therefore, $\left\{\tilde{y}_{n}\right\}$ is bounded. We may suppose that $\tilde{y}_{n} \rightarrow \tilde{y} \in X$. Then we get the sequence $\left\{t_{n}\right\} \subset \mathbb{R}_{+},\left\{\left(p_{n}, y_{n}\right)\right\} \subset P \times X$ such that $y_{n} \in K\left(p_{n}\right)$ for any $n \in \mathbb{N}$ and

$$
p_{n} \rightarrow \bar{p}, \quad \tilde{t}_{n}\left(p_{n}-p, y_{n}-\bar{y}\right) \rightarrow(p, y)
$$

which means that $y \in D^{S} K(\bar{p}, \bar{y})(p)$. By (12),

$$
\bar{y}+\frac{1}{t_{n}} \tilde{y}_{n} \in K\left(\bar{p}+\frac{1}{t_{n}} \tilde{p}_{n}\right), \quad z_{n}=f\left(\bar{p}+\frac{1}{t_{n}} \tilde{p}_{n}, \bar{x}+\frac{1}{t_{n}} \tilde{x}_{n}, \bar{y}+\frac{1}{t_{n}} \tilde{y}_{n}\right) .
$$


Since $K$ is compact and $f$ is continuous, we get $\bar{y} \in K(\bar{p})$ and $f(\bar{p}, \bar{x}, \bar{y})=0$. Using the Fréchet differentiability of $f$ at $(\bar{p}, \bar{x}, \bar{y})$, we have

$$
z=\lim _{n \rightarrow \infty} t_{n} z_{n}=\lim _{n \rightarrow \infty} \frac{f\left(\bar{p}+\frac{1}{t_{n}} \tilde{p}_{n}, \bar{x}+\frac{1}{t_{n}} \tilde{x}_{n}, \bar{y}+\frac{1}{t_{n}} \tilde{y}_{n}\right)}{\frac{1}{t_{n}}}=\nabla f(\bar{p}, \bar{x}, \bar{y})(p, x, \tilde{y}) .
$$

Therefore, (8) has been established for $p \neq 0$.

Next, we give formulae for inner and outer evaluating of the $\mathrm{S}$-derivative of the perturbation map $V$ in parameterized vector equilibrium problems by using the $\mathrm{S}$-derivative of the constraint mapping $K$ and the Fréchet derivative of the objective function $f$.

Theorem 4.1 Let $\bar{p} \in P, \bar{x} \in X$ and $\bar{y} \in \Omega=\{y \in K(\bar{p}) \mid f(\bar{p}, \bar{x}, y)=0\}$. Assume that $f$ is continuous and Fréchet differentiable at $(\bar{p}, \bar{x}, \bar{y})$ and $G$ has the domination property around $(\bar{p}, \bar{x})$. Assume further that one of the following conditions is satisfied:

(i) $D^{S} K(\bar{p}, \bar{y})(0)=\{0\}$.

(ii) $D^{S} G(\bar{p}, \bar{x}, 0)(0,0) \cap(-C)=\{0\}$ or $G$ is convex at $(p, x) \in U$, with $U$ being a neighborhood of $(\bar{p}, \bar{x})$.

Then, for any $(p, x) \in P \times X$, we have

$$
D^{S} V(\bar{p}, \bar{x}, 0)(p, x) \supset \min _{C} \bigcup_{\bar{y} \in \Omega}\left\{\nabla f(\bar{p}, \bar{x}, \bar{y})(p, x, y) \mid y \in D^{S} K(\bar{p}, \bar{y})(p)\right\} .
$$

Proof Using Lemma 4.1 and Theorem 3.1, we obtain the above results.

Theorem 4.2 Let $\bar{p} \in P, \bar{x} \in X$ and $\bar{y} \in \Omega=\{y \in K(\bar{p}) \mid f(\bar{p}, \bar{x}, y)=0\}$. Assume that $f$ is continuous and Fréchet differentiable at $(\bar{p}, \bar{x}, \bar{y})$. Furthermore, (5) and (6) hold true. Then, for any $(p, x) \in P \times X$, one has

$$
D^{S} V(\bar{p}, \bar{x}, 0)(p, x) \subset \min _{\operatorname{int} C} \bigcup_{\bar{y} \in \Omega}\left\{\nabla f(\bar{p}, \bar{x}, \bar{y})(p, x, y) \mid y \in D^{S} K(\bar{p}, \bar{y})(p)\right\} .
$$

Proof The proof follows from Lemma 4.1 and Theorem 3.2.

Remark 4.1 The results on sensitivity analysis for vector equilibrium problems in Theorem 4.1 and Theorem 4.2 are new because we use the $S$-derivative of a set-valued mapping, while the other authors $[24,25,31]$ used a contingent derivative and an adaptive subgradient for computing set-valued derivative formulae on some vector optimization problems.

Example 4.1 Let $T=[0,1] \cup\{-1\} \cup\{2\}, P=\mathbb{R}, X=\mathbb{R}^{2}, C=\mathbb{R}_{+}^{2} \cdot f: \mathbb{R} \times \mathbb{R}^{2} \times \mathbb{R}^{2} \rightarrow \mathbb{R}^{2}$, and $g_{t}: \mathbb{R} \times \mathbb{R}^{2} \rightarrow \mathbb{R}, t \in T$ is defined as

$$
\begin{aligned}
& f(p, x, y):=\left(2 p+x_{1}+y_{1}, x_{2}+y_{2}\right), \quad \forall x=\left(x_{1}, x_{2}\right), y=\left(y_{1}, y_{2}\right) \in \mathbb{R}^{2}, \forall p \in P . \\
& g_{t}(p, x):=t p-t x_{1}-(1-t) x_{2}, \quad \forall x=\left(x_{1}, x_{2}\right) \in \mathbb{R}^{2}, \forall p \in P .
\end{aligned}
$$

Reference [19], for all $(p, x) \in P \times X$, we get

$$
K(p)=\left\{x \in \mathbb{R}^{2} \mid-p+x_{1}-2 x_{2} \leq 0,2 p-2 x_{1}+x_{2} \leq 0\right\},
$$




$$
G(p, x)=\left\{z \in \mathbb{R}^{2} \mid-3 p+\left(2 x_{2}-x_{1}\right)+\left(z_{1}-2 z_{2}\right) \leq 0,6 p+\left(2 x_{1}-x_{2}\right)+\left(z_{2}-2 z_{1}\right) \leq 0\right\} .
$$

In particular, for $\bar{p}:=0$ and $\bar{x}=(0,0)$,

$$
\begin{aligned}
& K(\bar{p})=\left\{x \in \mathbb{R}^{2} \mid x_{1}-2 x_{2} \leq 0,-2 x_{1}+x_{2} \leq 0\right\}, \\
& G(\bar{p}, \bar{x})=\left\{z \in \mathbb{R}^{2} \mid\left(2 y_{2}-y_{1}\right)+\left(z_{1}-2 z_{2}\right) \leq 0,\left(2 y_{1}-y_{2}\right)+\left(z_{2}-2 z_{1}\right) \leq 0\right\},
\end{aligned}
$$

and thus $\bar{y}:=(0,0) \in K(\bar{p})$ and $\bar{z}:=f(\bar{p}, \bar{x}, \bar{y})=(0,0) \in V(\bar{p}, \bar{x})$. It is easy to check that $G$ is convex and $G$ has the domination property. By simple computation, one has

$$
D^{S} K(\bar{p}, \bar{y})(p)=\left\{y \in \mathbb{R}^{2} \mid-p+y_{1}-2 y_{2} \leq 0,2 p-2 y_{1}+y_{2} \leq 0\right\}, \quad \forall p \in P .
$$

Hence,

$$
\begin{aligned}
\{\nabla & \left.f(\bar{p}, \bar{x}, \bar{y})(p, x, y) \mid y \in D^{S} K(\bar{p}, \bar{y})(p)\right\} \\
= & \left\{\nabla_{p} f(\bar{p}, \bar{x}, \bar{y})(p)+\nabla_{x} f(\bar{p}, \bar{x}, \bar{y})(x)+\nabla_{y} f(\bar{p}, \bar{x}, \bar{y})(y) \mid y \in D^{S} K(\bar{p}, \bar{y})(p)\right\} \\
= & \left\{z \in \mathbb{R}^{2} \mid-3 p+\left(2 x_{2}-x_{1}\right)+\left(z_{1}-2 z_{2}\right) \leq 0,\right. \\
& \left.6 p+\left(2 x_{1}-x_{2}\right)+\left(z_{2}-2 z_{1}\right) \leq 0\right\} .
\end{aligned}
$$

Moreover, for all $(p, x) \times P \times X$,

$$
\begin{aligned}
D^{S} G(\bar{p}, \bar{x}, \bar{z})(p, x)= & \left\{z \in \mathbb{R}^{2} \mid-3 p+\left(2 x_{2}-x_{1}\right)+\left(z_{1}-2 z_{2}\right) \leq 0,\right. \\
& \left.6 p+\left(2 x_{1}-x_{2}\right)+\left(z_{2}-2 z_{1}\right) \leq 0\right\} .
\end{aligned}
$$

Therefore, (8) is valid. Finally, using Theorem 4.1 and Theorem 4.2, we have

$$
D^{S} V(\bar{p}, \bar{x}, \bar{z})(p, x) \subset\{(4 p, 0)\}, \quad D^{S} V(\bar{p}, \bar{x}, \bar{z})(p, x) \supset\{(4 p, 0)\}, \quad \forall(p, x) \in P \times X .
$$

This shows that

$$
D^{S} V(\bar{p}, \bar{x}, \bar{z})(p, x)=\{(4 p, 0)\}, \quad \forall(p, x) \in P \times X .
$$

\section{S-derivative of solution mapping for parametric vector equilibrium problems}

By the definition of gap functions for the parametric vector equilibrium problems and the parametric vector optimization problems, it is not difficult to find that the solution sets of these optimization problems can be unified via the following parametric variational system:

$$
E(p)=\{x \in K(p) \mid r(p, x) \in Q(p, x)\}
$$

where $Q: P \times X \rightarrow 2^{Y}, K: P \rightarrow 2^{X}$ are set-valued mappings and $r: P \times X \rightarrow Y$ is a vectorvalued mapping.

As we all known, $r(p, x) \in Q(p, x)$ can be regarded as a special class of generalized equations, which was first proposed by [32] and has been widely studied by scholars, because 
it provides a unified framework for the optimal solution of many optimization problems, such as mathematical programming, complementarity problems, variational inequalities, optimal control, multi-objective optimization problems, vectors variational inequalities, vector equilibrium problems, and so on.

Using the results of Sect. 3, if $r(p, x)=0, Q(p, x)=V(p, x)$, then (15) becomes a solution mapping of the parametric vector equilibrium problem

$$
E(p)=\{x \in K(p) \mid 0 \in V(p, x)\} .
$$

Therefore, we first investigate the formulae of $S$-derivative for the parametric variational systems in order to obtain the formulae of $S$-derivative for the parametric vector equilibrium problems.

Definition 5.1 ([32]) The set-valued mapping $Q$ is said to be like-Lipschitz at $(\bar{p}, \bar{x}, \bar{z}) \in$ gph $Q$ iff there exist a constant $\gamma>0$ and a neighborhood $U$ at $(\bar{p}, \bar{x})$ and a neighborhood $V$ at $\bar{z}$ such that

$$
Q\left(p_{1}, x_{1}\right) \cap V \subset Q\left(p_{2}, x_{2}\right)+\gamma\left(\left\|p_{1}-p_{2}\right\|+\left\|x_{1}-x_{2}\right\|\right), \quad \forall\left(p_{1}, x_{1}\right),\left(p_{2}, x_{2}\right) \in U .
$$

Definition 5.2 ([25]) The set-valued mapping $E$ is said to be Robinson metric regular relative to the set-valued mapping $K$ at $(\bar{p}, \bar{x}) \in \operatorname{gph} E$ iff there exist a constant $\mu, \gamma>0$ and a neighborhood $U$ at $(\bar{p}, \bar{x})$ satisfying $d(r(p, x), Q(p, x))<\gamma$, we have

$$
d(x, E(p)) \leq \mu d(r(p, x), Q(p, x)) .
$$

Theorem 5.1 Let $(\bar{p}, \bar{x}) \in \operatorname{gph} E, \bar{z}=r(\bar{p}, \bar{x})$. Assume that $r$ is Fréchet differentiable at $(\bar{p}, \bar{x})$, $E$ is Robinson metric regular relative to the set-valued mapping $K$ at $(\bar{p}, \bar{x}) \in \operatorname{gph} E$ and $Q$ is like-Lipschitz at $(\bar{p}, \bar{x}, \bar{z}) \in \operatorname{gph} Q$. Assume further that (7) holds true. Then, for any $p \in P$, we have

$$
D^{S} E(\bar{p}, \bar{x})(p)=\left\{x \in D^{S} K(\bar{p}, \bar{x})(p) \mid \nabla r(\bar{p}, \bar{x})(p, x) \in D^{S} Q(\bar{p}, \bar{x}, \bar{z})(p, x)\right\} .
$$

Proof Let us first justify that (7) leads to the following:

$$
D^{S} Q(\bar{p}, \bar{x}, \bar{z})(0,0)=\{0\}
$$

Assume the contrary of (17), then there is $z_{0} \in D^{S} Q(\bar{p}, \bar{x}, \bar{z})(0,0) \backslash\{0\}$. Hence, there are sequences $\left\{t_{n}\right\} \subset \mathbb{R}_{+},\left\{\left(p_{n}, x_{n}\right)\right\} \subset P \times X$ and $\left\{z_{n}\right\} \subset Y$ such that

$$
z_{n}=r\left(p_{n}, x_{n}\right) \in Q\left(p_{n}, x_{n}\right), \quad\left(p_{n}, x_{n}\right) \rightarrow(\bar{p}, \bar{x}), \quad t_{n}\left(p_{n}-\bar{p}, x_{n}-\bar{x}, z_{n}-\bar{z}\right) \rightarrow\left(0,0, z_{0}\right) .
$$

Thus, if $\left(p_{n}, x_{n}\right)=(\bar{p}, \bar{x})$ for all $n \in \mathbb{N}$, we take $t_{n} \in\left(0, \min \left\{\frac{1}{n}, \frac{\left\|z_{n}-\bar{z}\right\|}{n}\right\}\right)$, else $t_{n}=\max \left\{\| p_{n}-\right.$ $\left.\bar{p}\|,\| x_{n}-\bar{x} \|\right\}$. Then $t_{n} \rightarrow 0$ and $\left\|\frac{z_{n}-\bar{z}}{t_{n}}\right\| \rightarrow \infty$. Let

$$
\tilde{p}_{n}=\frac{p_{n}-\bar{p}}{t_{n}}, \quad \tilde{x}_{n}=\frac{x_{n}-\bar{x}}{t_{n}}, \quad \forall n \in \mathbb{N}
$$


Since $\left\|\tilde{p}_{n}\right\| \leq 1$ and $\left\|\tilde{x}_{n}\right\| \leq 1$ for all $n \in \mathbb{N}$, we may suppose that $\tilde{p}_{n} \rightarrow \tilde{p} \in P$ and $\tilde{x}_{n} \rightarrow$ $\tilde{x} \in X$. Since $r$ is Fréchet differentiable at $(\bar{p}, \bar{x})$, it holds that

$$
\lim _{n \rightarrow \infty} \frac{z_{n}-\bar{z}}{t_{n}}=\lim _{n \rightarrow \infty} \frac{r\left(\bar{p}+t_{n} \tilde{p}_{n}, \bar{x}+t_{n} \tilde{x}_{n}\right)-r(\bar{p}, \bar{x})}{t_{n}}=\operatorname{\nabla r}(\bar{p}, \bar{x})(\tilde{p}, \tilde{x}) .
$$

which contradicts $\left\|\frac{z_{n}-\bar{z}}{t_{n}}\right\| \rightarrow \infty$. Combining this with (7) and (17), we have (16) holds true for $p=0$.

Hence, it is enough to prove (16) in the case $p \neq 0$. Take any $p \in P \backslash\{0\}$ and $x \in$ $D^{S} E(\bar{p}, \bar{x})(p)$. Then there are $\left\{t_{n}\right\} \subset \mathbb{R}_{+},\left\{p_{n}\right\} \subset P$ and $x_{n} \in E\left(p_{n}\right)$ for all $n \in \mathbb{N}$ such that

$$
p_{n} \rightarrow \bar{p}, \quad t_{n}\left(p_{n}-\bar{p}, x_{n}-\bar{x}\right) \rightarrow(p, x)
$$

By $x_{n} \in E\left(p_{n}\right)$, there exists $\left\{x_{n}\right\} \subset X$ such that

$$
x_{n} \in K\left(p_{n}\right), \quad z_{n}=r\left(p_{n}, x_{n}\right) \in Q\left(p_{n}, x_{n}\right) .
$$

This means that there are $\left\{t_{n}\right\} \subset R_{+},\left\{p_{n}\right\} \subset P, x_{n} \in K\left(p_{n}\right)$ such that

$$
p_{n} \rightarrow \bar{p}, \quad t_{n}\left(p_{n}-\bar{p}, x_{n}-\bar{x}\right) \rightarrow(p, x)
$$

Thus, $x \in D^{S} K(\bar{p}, \bar{x})(p)$. Since $p_{n} \rightarrow \bar{p}, \tilde{p}_{n}=t_{n}\left(p_{n}-\bar{p}\right) \rightarrow p$, and $p \neq 0, t_{n}$ is unbounded. We may suppose that $t_{n} \rightarrow \infty$. Set $\tilde{x}_{n}=t_{n}\left(x_{n}-\bar{x}\right)$. By (19), we have

$$
r\left(\bar{p}+\frac{1}{t_{n}} \tilde{p}_{n}, \bar{x}+\frac{1}{t_{n}} \tilde{x}_{n}\right) \in Q\left(\bar{p}+\frac{1}{t_{n}} \tilde{p}_{n}, \bar{x}+\frac{1}{t_{n}} \tilde{x}_{n}\right) .
$$

Using (18) and $r$ is Fréchet differentiable at $(\bar{p}, \bar{x})$, it holds that

$$
\lim _{n \rightarrow \infty} t_{n}\left(z_{n}-\bar{z}\right)=\lim _{n \rightarrow \infty} \frac{r\left(\bar{p}+\frac{1}{t_{n}} \tilde{p}_{n}, \bar{x}+\frac{1}{t_{n}} \tilde{x}_{n}\right)-r(\bar{p}, \bar{x})}{\frac{1}{t_{n}}}=\operatorname{\nabla r}(\bar{p}, \bar{x})(p, x) .
$$

Thus, there are $\left\{t_{n}\right\} \subset \mathbb{R}_{+},\left\{\left(p_{n}, x_{n}\right)\right\} \subset P \times X$ and $z_{n} \in Q\left(p_{n}, x_{n}\right)$ such that

$$
\left(p_{n}, x_{n}\right) \rightarrow(\bar{p}, \bar{x}), \quad t_{n}\left(p_{n}-\bar{p}, x_{n}-\bar{x}, z_{n}-\bar{z}\right) \rightarrow(p, x, \nabla r(\bar{p}, \bar{x})(p, x)) .
$$

This means that $\nabla r(\bar{p}, \bar{x})(p, x) \in D^{S} Q(\bar{p}, \bar{x}, \bar{z})(p, x)$.

We now verify the reverse inclusion. For each $p \in P \backslash\{0\}$, take any $y \in D^{S} K(\bar{p}, \bar{x})(p)$ and $z=\nabla r(\bar{p}, \bar{x})(p, x) \in D^{S} Q(\bar{p}, \bar{x}, \bar{z})(p, x)$. Then there exist sequences $\left\{t_{n}\right\} \subset \mathbb{R}_{+},\left\{\left(p_{n}, x_{n}\right)\right\} \subset P \times$ $X$ and $z_{n} \in Q\left(p_{n}, x_{n}\right)$ for all $n \in \mathbb{N}$ such that

$$
\left(p_{n}, x_{n}\right) \rightarrow(\bar{p}, \bar{x}), \quad t_{n}\left(p_{n}-\bar{p}, x_{n}-\bar{x}, z_{n}-\bar{z}\right) \rightarrow(p, x, z) .
$$

Since $p_{n} \rightarrow \bar{p}, \tilde{p}_{n}:=t_{n}\left(p_{n}-\bar{p}\right) \rightarrow p \neq 0$, it shows that $\left\{t_{n}\right\}$ is unbounded. Without loss of generality, one may suppose that $t_{n} \rightarrow \infty$. Put

$$
\tilde{t}_{n}=\frac{1}{t_{n}}, \quad p_{n}=\bar{p}+\tilde{t}_{n} \tilde{p}_{n}, \quad \tilde{x}_{n}=t_{n}\left(x_{n}-\bar{x}\right), \quad \tilde{z}_{n}=t_{n}\left(z_{n}-\bar{z}\right) .
$$


Then we have

$$
\tilde{x}_{n} \rightarrow x, \tilde{z}_{n} \rightarrow z, \quad x_{n}=\bar{x}+\tilde{t}_{n} \tilde{x}_{n}, \quad z_{n}=\bar{z}+\tilde{t}_{n} \tilde{z}_{n} .
$$

Since $z_{n} \in Q\left(p_{n}, x_{n}\right)$, we have

$$
\bar{z}+\tilde{t}_{n} \tilde{z}_{n} \in Q\left(\bar{p}+\tilde{t}_{n} \tilde{p}_{n}, \bar{x}+\tilde{t}_{n} \tilde{x}_{n}\right)
$$

By $x \in D^{S} K(\bar{p}, \bar{x})(p)$, there exist $\left\{t_{n}\right\} \subset \mathbb{R}_{+},\left\{p_{n}^{\prime}\right\} \subset P$ and $x_{n}^{\prime} \in K\left(p_{n}^{\prime}\right)$ such that

$$
p_{n}^{\prime} \rightarrow \bar{p}, \quad t_{n}\left(p_{n}^{\prime}-\bar{p}, x_{n}^{\prime}-\bar{x}\right) \rightarrow(p, x) .
$$

Set

$$
\tilde{p}_{n}^{\prime}=t_{n}\left(p_{n}^{\prime}-\bar{p}\right), \quad \tilde{x}_{n}^{\prime}=t_{n}\left(x_{n}^{\prime}-\bar{x}\right) .
$$

Then we have

$$
\tilde{p}_{n}^{\prime} \rightarrow p, \tilde{x}_{n}^{\prime} \rightarrow x, \quad p_{n}^{\prime}=\bar{p}+\tilde{t}_{n} \tilde{p}_{n}^{\prime}, \quad x_{n}^{\prime}=\bar{x}+\tilde{t}_{n} \tilde{x}_{n}^{\prime} .
$$

Combining with $x_{n}^{\prime} \in K\left(p_{n}^{\prime}\right)$, we get $\bar{x}+\tilde{t}_{n} \tilde{x}_{n}^{\prime} \in K\left(\bar{p}+\tilde{t}_{n} \tilde{p}_{n}^{\prime}\right)$. Since $Q$ is like-Lipschitz at $(\bar{p}, \bar{x}, \bar{z}) \in \operatorname{gph} Q$, there exist constants $a_{1}, a_{2}>0$ and $\delta>0$ such that $b_{n} \in B(0, \delta)$ and $z_{n}^{\prime} \in$ $Q\left(\bar{p}+\tilde{t}_{n} \tilde{p}_{n}, \bar{x}+\tilde{t}_{n} \tilde{x}_{n}\right)$ for all $n \geq n_{0}$ satisfy

$$
\bar{z}+\tilde{t}_{n} \tilde{z}_{n}=z_{n}^{\prime}+t_{n}\left(a_{1}\left\|p_{n}-p_{n}^{\prime}\right\|+a_{2}\left\|x_{n}-x_{n}^{\prime}\right\|\right) b_{n} .
$$

Since $E$ is Robinson metric regular relative to $K$ at $(\bar{p}, \bar{x}) \in \operatorname{gph} E$, there are $\mu>0, \gamma>0$, a neighborhood $U$ of $(\bar{p}, \bar{x})$ satisfies $d(r(p, x), Q(p, x))<\gamma$, we have

$$
d(x, E(p)) \leq \mu d(r(p, x), Q(p, x)) .
$$

Since $r$ is Fréchet differentiable at $(\bar{p}, \bar{x})$, then there is $n_{0} \in \mathbb{N}$ such that, for all $n \geq n_{0}$, we have

$$
r\left(\bar{p}+\tilde{t}_{n} \tilde{p}_{n}^{\prime}, \bar{x}+\tilde{t}_{n} \tilde{x}_{n}^{\prime}\right)=r(\bar{p}, \bar{x})+\tilde{t}_{n}\left(\nabla r(\bar{p}, \bar{x})\left(\tilde{p}_{n}, \tilde{x}_{n}\right)+\frac{o\left(\left\|t_{n}\left(p_{n}^{\prime}, x_{n}^{\prime}\right)\right\|\right)}{t_{n}}\right) .
$$

Hence,

$$
\begin{aligned}
& d\left(r\left(\bar{p}+\tilde{t}_{n} \tilde{p}_{n}^{\prime}, \bar{x}+\tilde{t}_{n} \tilde{x}_{n}^{\prime}\right), Q\left(\bar{p}+\tilde{t}_{n} \tilde{p}_{n}^{\prime}, \bar{x}+\tilde{t}_{n} \tilde{x}_{n}^{\prime}\right)\right) \\
& \quad \leq\left\|r\left(\bar{p}+\tilde{t}_{n} \tilde{p}_{n}^{\prime}, \bar{x}+\tilde{t}_{n} \tilde{x}_{n}^{\prime}\right)-\bar{z}-\tilde{t}_{n} \tilde{z}_{n}+\tilde{t}_{n}\left(a_{1}\left\|p_{n}-p_{n}^{\prime}\right\|+a_{2}\left\|x_{n}-x_{n}^{\prime}\right\|\right) b_{n}\right\|=\tilde{t}_{n} \tilde{\beta}(n),
\end{aligned}
$$

where

$$
\beta(n)=\left\|\nabla r(\bar{p}, \bar{x})\left(\tilde{p}_{n}, \tilde{x}_{n}\right)+\frac{o\left(\left\|\tilde{t}_{n}\left(p_{n}^{\prime}, x_{n}^{\prime}\right)\right\|\right)}{\tilde{t}_{n}}-\tilde{z}_{n}+\left(a_{1}\left\|p_{n}-p_{n}^{\prime}\right\|+a_{2}\left\|x_{n}-x_{n}^{\prime}\right\|\right) b_{n}\right\| .
$$


By (20), we have

$$
d\left(\bar{x}+\tilde{t}_{n} \tilde{x}_{n}^{\prime}, E\left(\bar{p}+\tilde{t}_{n} \tilde{p}_{n}^{\prime}\right)\right) \leq \mu d\left(r\left(\bar{p}+\tilde{t}_{n} \tilde{p}_{n}^{\prime}, \bar{x}+\tilde{t}_{n} \tilde{x}_{n}^{\prime}\right), Q\left(\bar{p}+\tilde{t}_{n} \tilde{p}_{n}^{\prime}, \bar{x}+\tilde{t}_{n} \tilde{x}_{n}^{\prime}\right)\right) \leq \mu \tilde{t}_{n} \tilde{\beta}(n) .
$$

So there is $\bar{x}+\tilde{t}_{n} \tilde{x}_{n}^{\prime} \in E\left(\bar{p}+\tilde{t}_{n} \tilde{p}_{n}^{\prime}\right)+\left(\mu \tilde{t}_{n} \tilde{\beta}(n)+\tilde{t}_{n}^{2}\right) B(0, \delta)$, which means that there exists $b_{n}^{\prime} \in B(0, \delta)$ such that

$$
x_{n}^{\prime \prime}=\bar{x}+\tilde{t}_{n} \tilde{x}_{n}^{\prime}-\tilde{t}_{n}\left(\mu \tilde{\beta}(n)+\tilde{t}_{n}\right) b_{n}^{\prime}=x_{n}^{\prime}-\tilde{t}_{n}\left(\mu \tilde{\beta}(n)+\tilde{t}_{n}\right) b_{n}^{\prime} \in E\left(\bar{p}+\tilde{t}_{n} \tilde{p}_{n}^{\prime}\right) .
$$

Since $\left(\mu \tilde{\beta}(n)+\tilde{t}_{n}\right)\left\|b_{n}^{\prime}\right\|$ converges to 0 , this means that there are sequences $\left\{t_{n}\right\} \subset \mathbb{R}_{+}$, $\left\{p_{n}^{\prime}\right\} \subset P$ and $x_{n}^{\prime \prime} \in E\left(p_{n}^{\prime}\right)$ such that

$$
p_{n}^{\prime} \rightarrow \bar{p}, t_{n}\left(p_{n}^{\prime}-\bar{p}, x_{n}^{\prime \prime}-\bar{x}\right)=t_{n}\left(p_{n}^{\prime}-\bar{p}, x_{n}^{\prime}-\bar{x}-\left(\mu \tilde{\beta}(n)+\tilde{t}_{n}\right) b^{\prime}\right) \rightarrow(p, x) .
$$

This shows that $x \in D^{S} E(\bar{p}, \bar{x})(p)$.

Corollary 5.1 Assume that all conditions in Theorem 4.1 and Theorem 5.1 are satisfied. Then, for any $p \in P$, we have

$$
D^{S} E(\bar{p}, \bar{x})(p) \supset\left\{x \in D^{S} K(\bar{p}, \bar{x})(p) \mid \nabla f(\bar{p}, \bar{x}, \bar{y})(p, x, y) \notin-C \backslash\{0\}, y \in D^{S} K(\bar{p}, \bar{y})(p)\right\} .
$$

Corollary 5.2 Assume that all conditions in Theorem 4.2 and Theorem 5.1 are satisfied. Then, for any $p \in P$, we have

$$
D^{S} E(\bar{p}, \bar{x})(p) \subset\left\{x \in D^{S} K(\bar{p}, \bar{x})(p) \mid \nabla f(\bar{p}, \bar{x}, \bar{y})(p, x, y) \notin-\operatorname{int} C, y \in D^{S} K(\bar{p}, \bar{y})(p)\right\} .
$$

\section{Conclusion}

In this paper, we have dealt with the sensitivity analysis on vector equilibrium problems. $S$-derivative estimations on a perturbed mapping for the parametric vector equilibrium problem are given via a set-valued gap function. Moreover, we derive $S$-derivative estimations of a solutions mapping of the parametric vector equilibrium problems by $S$ derivative estimations of a kind of the parametric variational system. However, how to investigate the sensitivity analysis of the parametric vector equilibrium problems by using other set-valued derivatives is still an interesting problem.

\section{Acknowledgements}

The authors thank the editor and the reviewers for their helpful comments and suggestions, which have improved the presentation of the paper.

\section{Funding}

This research was supported by the National Natural Science Foundation of China (No.11761023), Support Plan for Science and Technology Top-notch Talents of Guizhou Higher Education Institutions (No. 2017[081]), and Natural Science Foundation of Guizhou Normal College (No. 2017BS009).

Availability of data and materials

Not applicable.

Competing interests

The authors declare that they have no competing interests. 


\section{Author details}

'Department of Mathematics and Computer, Guizhou Normal College, 550018, Guiyang, Guizhou, China. ${ }^{2}$ School of Mathematics and Statistics, Guizhou University, 550025, Guiyang, Guizhou, China.

\section{Publisher's Note}

Springer Nature remains neutral with regard to jurisdictional claims in published maps and institutional affiliations.

Received: 28 April 2021 Accepted: 12 July 2021 Published online: 26 July 2021

\section{References}

1. Clarke, F.H.: Optimization and Nonsmooth Analysis. Wiley, New York (1983)

2. Fiacco, A.V.: Introduction to Sensitivity and Stability Analysis in Nonlinear Programming. Academic Press, New York (1983)

3. Bonnans, J.F., Shapiro, A.: Perturbation Analysis of Optimization Problems. Springer, New York (2000)

4. Auslender, A., Teboulle, M.: Asymptotic Cones and Functions in Optimization and Variational Inequalities. Springer, New York (2003)

5. Borwein, J.M., Zhu, Q.J.: Techniques of Variational Analysis. Springer, New York (2005)

6. Mordukhovich, B.S.: Variational Analysis and Generalized Differentiation, Vol. I: Basic Theory, Vol. II: Applications. Springer, Berlin (2006)

7. Zhao, X., Kobis, M.A., Yao, Y., Yao, J.C.: A projected subgradient method for nondifferentiable quasiconvex multiobjective optimization problems. J. Optim. Theory Appl. 190, 82-107 (2021)

8. Zhu, L.J., Yao, Y., Postolache, M.: Projection methods with linesearch technique for pseudomonotone equilibrium problems and fixed point problems. UPB Sci. Bull., Ser. A 83, 3-14 (2021)

9. Ceng, L.C., Petrusel, A., Yao, J.C., Yao, Y.: Systems of variational inequalities with hierarchical variational inequality constraints for Lipschitzian pseudocontractions. Fixed Point Theory 20, 113-133 (2019)

10. Hebestreit, N.: Algorithms for monotone vector variational inequalities. J. Nonlinear Var. Anal. 4, 107-125 (2019)

11. Ju, X., Khan, S.A.: Well-posedness for bilevel vector equilibrium problems. Appl. Set-Valued Anal. Optim. 1, 29-38 (2019)

12. Cho, S.Y.: A convergence theorem for generalized mixed equilibrium problems and multivalued asymptotically nonexpansive mappings. J. Nonlinear Convex Anal. 21, 1017-1026 (2020)

13. Tanino, T.: Sensitivity analysis in multi-objective optimization. J. Optim. Theory Appl. 56, 479-499 (1988)

14. Tanino, T.: Stability and sensitivity analysis in convex vector optimization. SIAM J. Control Optim. 26, $521-536$ (1988)

15. Kuk, H., Tanino, T., Tanaka, M.: Sensitivity analysis in vector optimization. J. Optim. Theory Appl. 89, $713-730$ (1996)

16. Kuk, H., Tanino, T., Tanaka, M.: Sensitivity analysis in parametrized vector optimization. J. Math. Anal. Appl. 202 511-522 (1996)

17. Shi, D.S.: Contingent derivative of the perturbation map in multiobjective optimization. J. Optim. Theory Appl. 70, 385-396 (1991)

18. Shi, D.S.: Sensitivity analysis in convex vector optimization. J. Optim. Theory Appl. 77, 145-159 (1993)

19. Chuong, T.D.: Derivatives of the efficient point multifunction in parametric vector optimization problems. J. Optim. Theory Appl. 156, 247-265 (2013)

20. Aubin, J.P., Frankowska, H.: Set-Valued Analysis. Birkhäuser, Boston (1990)

21. Li, S.J., Yan, H., Chen, G.Y.: Differential and sensitivity properties of gap functions for vector variation inequalities. Math. Methods Oper. Res. 57, 377-391 (2003)

22. Meng, K.W., Li, S.J.: Differential and sensitivity properties of gap functions for Minty vector variational inequalities. J. Math. Anal. Appl. 337, 386-398 (2008)

23. Li, M.H., Li, S.J.: Second-order differential and sensitivity properties of weak vector variational inequalities. J. Optim. Theory Appl. 144, 76-87 (2010)

24. Li, M.H., Li, S.J., Fang, Z.M.: Stability and sensitivity analysis of solutions to weak vector variational inequalities. Set-Valued Var. Anal. 20,111-129(2012)

25. Li, S.J., Li, M.H.: Sensitivity analysis of parametric weak vector equilibrium problems. J. Math. Anal. Appl. 380, 354-362 (2011)

26. Chen, G.Y., Huang, X.X., Yang, X.Q.: Vector Optimization: Set-Valued and Variational Analysis. Lecture Notes in Economics and Mathematical Systems. Springer, Berlin (2005)

27. Sawaragi, Y., Nakayama, H., Tanino, T.: Theory of Multiobjective Optimization. Academic Press, New York (1985)

28. Farajzadeh, A.P., Plubteing, S.: On parametric implicit vector variational inequality problems. J. Inequal. Appl. 1, 1-7 (2014)

29. Farajzadeh, A.P., Shafie, A.: On system of vector quasi-equilibrium problems for multivalued mappings. Kragujev. J. Math. 42, 357-369 (2018)

30. Farajzadeh, A.P., Salahuddin: Sensitivity analysis for nonlinear set-valued variational equations in Banach framework. J. Funct. Spaces Appl. 2013, Article ID 258543 (2013)

31. Nimana, N., Farajzadeh, A.P., Petrot, N.: Adaptive subgradient method for the split quasi-convex feasibility problems. Optimization 65, 1885-1898 (2016)

32. Robinson, S.M.: Stability theory for systems of inequalities, I. Differentiable nonlinear systems. SIAM J. Numer. Anal. 65 497-513 (1976) 